\title{
ON THE MACROSCOPIC THEORY OF VAN DER WAALS FORCES
}

\author{
N.G.VAN KAMPEN, B.R.A. NIJBOER and K.SCHRAM \\ Instituut voor theoretische fysica, Rijksuniversiteit Utrecht, The Netherlands
}

Received 24 January 1968

\begin{abstract}
A simple macroscopic derivation is given of the non-retarded Van der Waals interaction between two semi-infinite dielectric media.
\end{abstract}

Neutral macroscopic bodies, when placed at a distance of the order of $1000 \AA$, attract each other as a result of the Van der Waals forces between their atoms. Lifshitz [1] has developed a macroscopic theory for this phenomenon by introducing fluctuating terms in the Maxwell equations for a dielectric medium. For the interaction energy per unit area at $T=0$ between two semi-infinite media a distance $d$ apart Lifshitz finds in the case that $d \ll \lambda$ ( $\lambda$ is the principal absorption wave length of the material)

$$
U(d)=-\frac{\hbar}{32 \pi^{2} d^{2}} \int_{0}^{\infty} \mathrm{d} \omega \int_{0}^{\infty} \mathrm{d} x x^{2}\left\{\left(\frac{\epsilon(\mathrm{i} \omega)+1}{\epsilon(\mathrm{i} \omega)-1}\right)^{2} \mathrm{e}^{x}-1\right\}^{-1},
$$

where $\epsilon(\omega)$ is the frequency dependent dielectric constant of the medium.

Starting from the Drude-Lorentz model of an atom (harmonic oscillator model) Renne and Nijboer [2] gave an atomistic derivation of the non-retarded interaction between one atom and a semi-infinite medium by summing two-particle, three-particle, etc., interactions. Their derivation can be extended to the case of two semi-infinite media and for arbitrary atoms [3]. In this note we will give a simple alternative macroscopic derivation of eq. (1), which can easily be generalized to more complicated configurations.

Consider two semi-infinite dielectric media a distance $d$ apart. We look for those solutions of the equations of electrostatics: $\operatorname{div} D=0$, curl $E=0$, which oscillate harmonically in time. It will be obvious that these equations can be satisfied by: $1^{\circ}$ : curl $E=0, \epsilon(\omega)=0$ (longitudinal bulk modes); $2^{\circ}: \operatorname{div} D=0, \epsilon(\omega)=\infty$ (transverse bulk modes); $3^{\circ}: \operatorname{div} E=0$, curl $E=0$ (surface modes). At $T=0$ the interaction between the media may be found from the sum of the zero point energies $\sum_{k} \frac{1}{2} \hbar \omega_{k}$ of all modes evaluated at a distance $d$ minus the corresponding sum for infinite $d$. This procedure, due to Casimir [4], who applied it to the case of ideal conductors in vacuo, can be justified in our case by considering a macroscopic Hamiltonian of the system which is equivalent to a sum of harmonic oscillator Hamiltonians with the frequencies $\omega_{k}$ as eigenfrequencies. A similar Hamiltonian was used by Hopfield [5].

Now the frequencies of the bulk modes $1^{\circ}$ and $2^{\circ}$ are evidently independent of the distance $d$. Hence for the evaluation of the Van der Waals interaction only the surface modes $3^{\circ}$ need be considered. Introducing the electrostatic potential $\varphi(x, y, z, t)$ by $E=-\operatorname{grad} \varphi$ we have to solve the equation $\nabla^{2} \varphi=0$, subject to the boundary conditions $\varphi$ and $\epsilon(\omega) \partial \varphi / \partial z$ continuous at $z=0$ and $z=d$ (the $z$-axis is chosen perpendicular to the plane-parallel boundaries of the media).

Assuming $\varphi$ to be of the form

$$
\varphi(x, y, z, t)=f(z) \exp (\mathrm{i} u x+\mathrm{i} v y-\mathrm{i} \omega t),
$$

we find for $f(z)$ the equation:

$$
f^{n}-r^{2} f=0 \text { with } \quad r^{2}=u^{2}+v^{2}
$$

with the solution

$$
f=A \mathrm{e}^{r z}(z \leqslant 0), \quad f=B \mathrm{e}^{-r z}(z \geqslant d), \quad f=C \mathrm{e}^{-r z}+D \mathrm{e}^{-r z}(0 \leqslant z \leqslant d) .
$$


The boundary conditions lead to the equation

$$
\left(\frac{\epsilon(\omega)+1}{\epsilon(\omega)-1}\right)^{2} \mathrm{e}^{2 r d}-1=0
$$

the positive roots of which (for $r$ ranging from 0 to $\infty$ ) determine the frequencies of the surface modes.

One can now apply the well-known theorem, that for a meromorphic function $g(\omega)$ the sum of the zeros minus the sum of the poles within a contour in the complex $\omega$-plane is given by the contour integral

$$
\frac{1}{2 \pi \mathbf{i}} \oint \omega \frac{\mathrm{d}}{\mathrm{d} \omega}(\ln g(\omega)) \mathrm{d} \omega .
$$

As a contour one takes a semi-circle in the right half of the complex $\omega$-plane, the radius of which is allowed to go to infinity. After some straightforward algebra one is then led to Lifshitz' formula (1).

In the particular case that $\epsilon(\omega)-1=$ const $/\left(\omega_{0}^{2}-\omega^{2}\right)$ one can solve eq. (2) for $\omega$ explicitly and evaluate the sum of eigenfrequencies directly without contour integration. The result is

$$
U(d)=-\frac{1}{8} \frac{\hbar \omega_{\mathrm{o}}}{\pi d^{2}}\left(\frac{\epsilon_{\mathrm{o}}+1}{2}\right)^{\frac{1}{2}} \sum_{n=1}^{\infty} \frac{a_{2 n}}{n^{2}}\left(\frac{\epsilon_{\mathrm{o}}-1}{\epsilon_{\mathrm{o}}+1}\right)^{2 n} .
$$

Here $\epsilon_{\mathrm{O}}$ is the static dielectric constant and

$$
a_{m}=(2 m):\left\{(2 m-1) m !^{2} 2^{2 m}\right\}^{-1} .
$$

This result may be of practical interest for the calculation of the Van der Waals interaction between media with only one resonance frequency.

Finally it will be obvious that the above calculation can be readily generalized to the evaluation of the interaction between two semi-infinite media with dielectric constants $\epsilon_{1}(\omega)$ and $\epsilon_{2}(\omega)$ respectively, separated by a dielectric of thickness $d$ and dielectric constant $\epsilon_{3}(\omega)$. The result is $(d \ll \lambda)$ :

$$
U(d)=-\frac{\hbar}{32 \pi^{2} d^{2}} \int_{0}^{\infty} \mathrm{d} \omega \int_{0}^{\infty} \mathrm{d} x x^{2}\left\{\frac{\left\{\epsilon_{1}(\mathrm{i} \omega)+\epsilon_{3}(\mathrm{i} \omega)\right\}\left\{\epsilon_{2}(\mathrm{i} \omega)+\epsilon_{3}(\mathrm{i} \omega)\right\}}{\left\{\epsilon_{1}(\mathrm{i} \omega)-\epsilon_{3}(\mathrm{i} \omega)\right\}\left\{\epsilon_{2}(\mathrm{i} \omega)-\epsilon_{3}(\mathrm{i} \omega)\right\}} \mathrm{e}^{x}-1\right\}^{-1},
$$

which coincides with an expression found by Dzyaloshinskii et al. [6] by applying Green functions techniques. A more detailed report will be published elsewhere. The case of retardation will also be considered there.

\section{References}

1. E. M. Lifshitz, Soviet Phys. JETP 2 (1956) 73;

cf. also L. Landau and E. M. Lifshitz, Electrodynamics of Continuous Media (Pergamon Press, Oxford, 1960) $\$ 90$.

2. M. J. Renne and B. R. A. Nijboer, Chem. Phys. Letters 1 (1967) 317.

3. Idem, to be published.

4. H. B. G. Casimir, Proc. Kon. Ned. Ak. Wet. 51 (1948) 793.

5. J. J. Hopfield, Phys. Rev. 112 (1958) 1555.

6. I. E. Dzyaloshinskii, E. M. Lifshitz and L.P. Pitaevskii, Soviet Phys. JETP 10 (1960) 161. 